\title{
Facile Solventless Synthesis of a Nylon-6,6/Silver Nanoparticles Composite and Its XPS Study
}

\author{
Raúl A. Morales-Luckie, ${ }^{1}$ Víctor Sánchez-Mendieta, ${ }^{2}$ Oscar Olea-Mejia, ${ }^{1}$ \\ Alfredo R. Vilchis-Nestor, ${ }^{1}$ Gustavo López-Téllez, ${ }^{1}$ Víctor Varela-Guerrero, ${ }^{1}$ \\ L. Huerta, ${ }^{3}$ and Jesús Arenas-Alatorre ${ }^{4}$ \\ ${ }^{1}$ Centro Conjunto de Investigación en Química Sustentable UAEM-UNAM (CCIQS), Facultad de Química, \\ Universidad Autónoma del Estado de México, Km. 14.5 de la carretera Toluca-Atlacomulco, 50200 San Cayetano, MEX, Mexico \\ ${ }^{2}$ Facultad de Química, Universidad Autónoma del Estado de México, Paseo Colón y Paseo Tollocan, 50120 Toluca, MEX, Mexico \\ ${ }^{3}$ Instituto de Investigaciones en Materiales, Universidad Nacional Autónoma de México, \\ Apartado Postal 70-360, 04510 México, DF, Mexico \\ ${ }^{4}$ Instituto de Física, Universidad Nacional Autónoma de México, Apartado Postal 20-364, 01000 México, DF, Mexico
}

Correspondence should be addressed to Víctor Sánchez-Mendieta; vsanchezm@uaemex.mx

Received 14 February 2013; Revised 22 April 2013; Accepted 9 May 2013

Academic Editor: Carmina Menchaca-Campos

Copyright (C) 2013 Raúl A. Morales-Luckie et al. This is an open access article distributed under the Creative Commons Attribution License, which permits unrestricted use, distribution, and reproduction in any medium, provided the original work is properly cited.

\begin{abstract}
Silver nanoparticles were synthesized and supported on thin nylon membranes by means of a simple method of impregnation and chemical reduction of Ag ions at ambient conditions. Particles of less than $10 \mathrm{~nm}$ were obtained using this methodology, in which the nylon fibers behave as constrained nanoreactors. Pores on nylon fibres along with oxygen and nitrogen from amide moieties in nylon provide effective sites for in situ reduction of silver ions and for the formation and stabilization of Ag nanoparticles. Transmission electron microscopy (TEM) analysis showed that silver nanoparticles are well dispersed throughout the nylon fibers. Furthermore, an interaction between nitrogen of amides moieties of nylon-6,6 and silver nanoparticles has been found by X-ray photoelectron spectroscopy (XPS).
\end{abstract}

\section{Introduction}

Research on the synthesis of mesoporous materials containing nanoparticles represents a fast-developing area of nanoscience and nanotechnology. This interest is stimulated by several possible application areas of these materials including catalytic [1], magnetic [2], and optoelectronic [3, 4]. Metal nanoparticles dispersed in polymeric matrixes have recently been the subject of intense study aiming to develop nanocomposite films [5-8]. General approaches for the synthesis and support of nanoparticles inside porous materials include impregnation [9] and deposition-precipitation [10]. A drawback of this nanocomposites is the difficulty to disperse nanoparticles in most systems [11]; hence one potential advantage of such metal/polymer systems is that the size and distribution of dispersed metal nanoparticles can be readily controlled based on the properties of the host polymer $[12,13]$.
Silver particles with a narrow size distribution have been produced upon reversible chemical transformation between metallic and oxide states in a titania matrix [14] and in a mesoporous silica which was grafted with hydrophobic $-\mathrm{Si}\left(\mathrm{CH}_{3}\right)_{3}$ groups at the pore surface [15].

Nylon is an electron-rich and polar synthetic polymer (polyamide) usually made from the monomers adipoyl chloride and hexamethylene diamine to form a linear molecular chain (Figure 1). Synthetic nylon membranes have a porous structure [16] and are composed of microfibrils that are interconnected forming a three-dimensional network. Such morphological features provide a unique reaction vessel for synthesizing and supporting metal nanoparticles, allowing enhanced access of guest molecules to catalytic centres, compared with nonporous films.

In this work, a facile synthesis of silver nanoparticles of less than $10 \mathrm{~nm}$ in diameter with a narrow size distribution, 

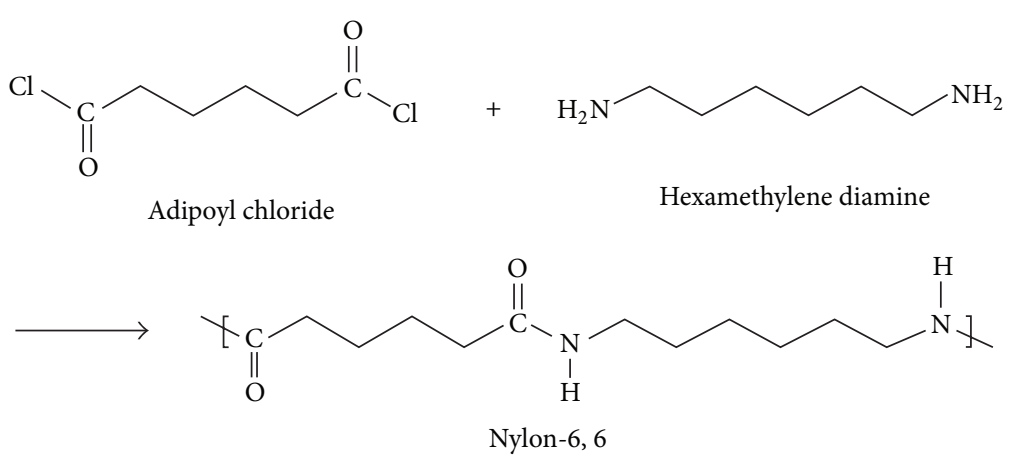

FIGURE 1: Characteristic synthesis of nylon-6,6 fibers.

using porous nylon fibers as unique nanoreactors to generate a nylon-6,6/silver nanoparticles composite, is reported.

\section{Experimental}

All chemical reagents were analytical grade and were used without further purification.

\subsection{Preparation of the Nylon-6,6/Ag Nanoparticles Composite.} In a typical experiment, nylon-6,6 membranes (Millipore Co., cat. number: GNWP02500; $150 \mu \mathrm{m}$ thick and $0.22 \mu \mathrm{m}$ pores average diameter) are impregnated with silver ions by immersing the membranes in $\mathrm{AgNO}_{3}(0.1 \mathrm{mM}, 0.5 \mathrm{mM}$, $1 \mathrm{mM}, 1.5 \mathrm{mM}$, and $10 \mathrm{mM}$; Aldrich) aqueous solution for $1 \mathrm{~min}$, followed by rinsing with ethanol for $30 \mathrm{~s}$. Then, nylon membranes are immersed in aqueous $\mathrm{NaBH}_{4}(2 \mathrm{mM}$; Aldrich), for reduction of silver ions, for $30 \mathrm{~min}$ and subsequently rinsed with deionized water for $1 \mathrm{~min}$. Finally, the specimen is vacuum-dried overnight at room temperature.

2.2. Characterization. UV-visible absorption spectra were recorded, using a nylon-6,6 membrane as the reference, on a Cary 5000 UV-Vis-NIR scanning spectrophotometer. Scanning electron microscopy (SEM) observations were performed on a Philips XL30 electron scanning microscope. Transmission electron microscopy (TEM) observations were carried out on a JEOL JEM-2010F instrument with a point resolution of $1.9 \AA$ and equipped with high-angle annular dark-field or $Z$-contrast detector. The nylon fibers with Ag nanoparticles were cut into pieces, dispersed in pure water, transferred onto a SiO-coated copper grid with disperser, dried in a vacuum overnight, and observed by TEM.

The measurements of lattice-fringe spacing and angles were made using digital image analysis of reciprocal space parameters, according to Akamatsu et al. [17]. Using this method the precision is $0.0001 \mathrm{~nm}$ for lattice spacing and $0.1^{\circ}$ for lattice plane angles. This analysis was carried out with the Digital Micrograph software, Oxford, UK.

X-ray photoelectron spectroscopy (XPS) was performed using an UHV system of VG Microtech ESCA3000 Multilab, with an $\mathrm{Al} \mathrm{K} \mathrm{K}_{\alpha} \mathrm{X}$-ray source $(1486.6 \mathrm{eV})$ and CLAM4MCD analyser. The surface of the sample was etched for $10 \mathrm{~s}$ with $3 \mathrm{kV} \mathrm{Ar}{ }^{+}$at $0.16 \mu \mathrm{A} / \mathrm{mm}^{2}$. The XPS spectrum was obtained at $55^{\circ}$ from the normal surface in the constant pass energy mode; the pass energies used were $50 \mathrm{eV}$ and $20 \mathrm{eV}$ for survey and high resolution, respectively. The peak positions were referenced to the Shirley background $\mathrm{Ag} 3 \mathrm{~d}_{5 / 2}$ photopeak at $368.21 \mathrm{eV}$ and $\mathrm{C} 1 \mathrm{~s}$ hydrocarbon groups in a binding energy of $285.00 \mathrm{eV}$. XPS spectra were fitted with the program SDP $\mathrm{v}$ 4.1 [18]. For the process of deconvolution the uncertainty in the binding energy was estimated to be $5 \%$ (i.e., $\pm 0.05 \mathrm{eV}$ ).

\section{Results and Discussion}

The membranes used in this work are composed of nylon-6,6 fibers in the micrometer range, as shown in Figure 2(a). The fibers surface is rough (Figure 2(b)) and has several pores of less than $100 \mathrm{~nm}$ in diameter (inset Figure 2(b)).

These pores may allow guest molecules to enter. Thus, when a nylon membrane is immersed in aqueous $\mathrm{AgNO}_{3}$, $\mathrm{Ag}^{+}$ions are readily impregnated into the nylon membrane fibers through the pores. Most of the incorporated $\mathrm{Ag}^{+}$ions are bound to nylon macromolecules probably via electrostatic (i.e., ion-dipole) interactions, because the electron-rich oxygen and nitrogen atoms of polar amides groups are expected to interact with positive metal ions. The posterior rinse with ethanol (ca. $30 \mathrm{~s}$ ) removes $\mathrm{Ag}^{+}$ions that were not anchored to nylon fibres. Figure 3 shows the pure nylon membrane and nylon membranes having Ag nanoparticles, using $0.5 \mathrm{mM}$, $1 \mathrm{mM}, 1.5 \mathrm{mM}$, and $10 \mathrm{mM} \mathrm{AgNO}_{3}$ solutions, respectively. As the concentration of nanoparticles increases, the membranes exhibit different colours from light yellow to yellow and finally yellow-brownish.

Energy-dispersive X-ray spectroscopy (EDX) spectrum in Figure 4, which was collected from the nylon-6,6/silver nanoparticles composite, clearly shows the presence of $\mathrm{Ag}$ signal peak, indicating the effectiveness of the electrostatic assembly of the particles on the surface of the nylon fibers. The yellowish colour of Ag-nylon composite membrane (Figure 3) also suggests the presence of Ag nanoparticles on the polymer surface.

UV-Vis spectra of the nylon-6,6/silver nanoparticles composites are shown in Figure 5. Because the nylon-6,6 membrane was used as the reference, the absorption spectra can be considered as the absorption of Ag species. Using $0.5 \mathrm{mM} \mathrm{AgNO}_{3}$ a narrow symmetrical absorption band is 


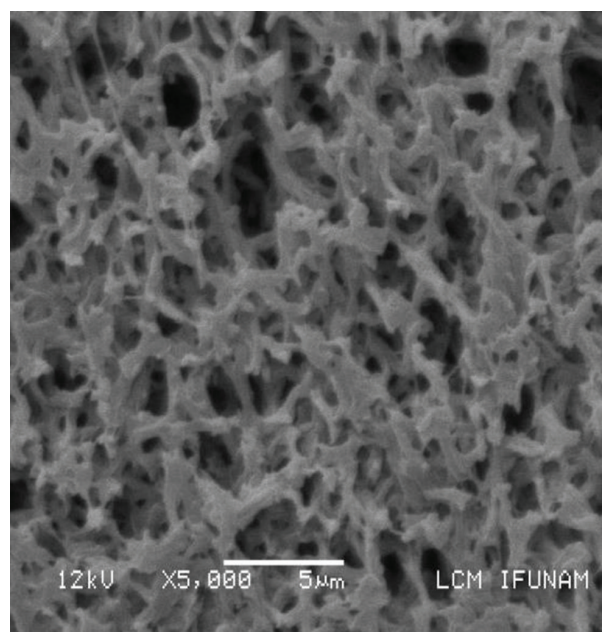

(a)

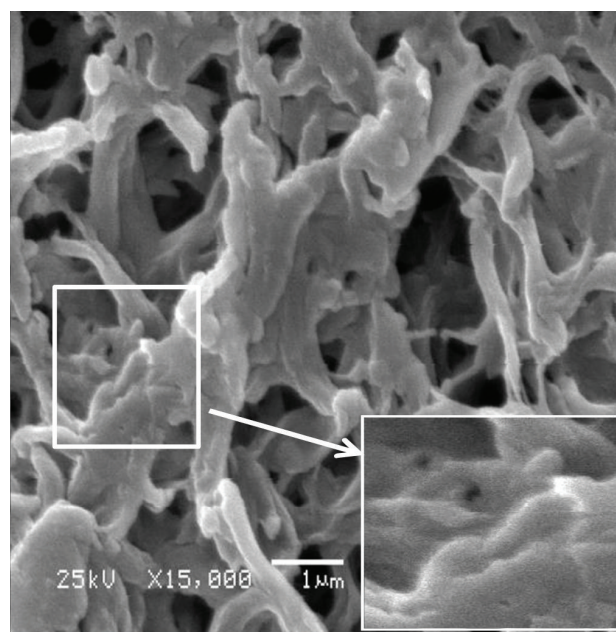

(b)

FIGURE 2: SEM image of the nylon-6,6 membrane.

Without Ag

$0.5 \mathrm{mM} \mathrm{Ag}$

$1 \mathrm{mM} \mathrm{Ag}$

$1.5 \mathrm{mM} \mathrm{Ag}$

$10 \mathrm{mM} \mathrm{Ag}$
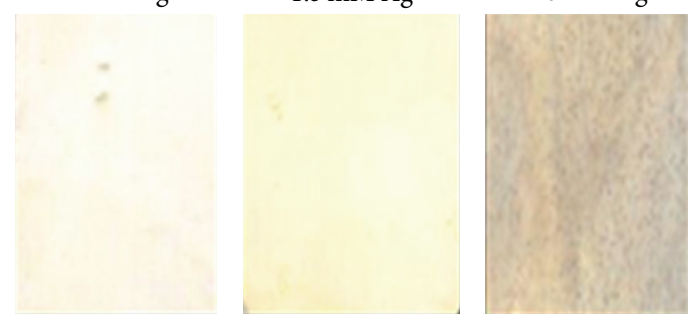

FIgURE 3: Nylon-6,6 without and with Ag nanoparticles formed at different $\mathrm{AgNO}_{3}$ concentrations.

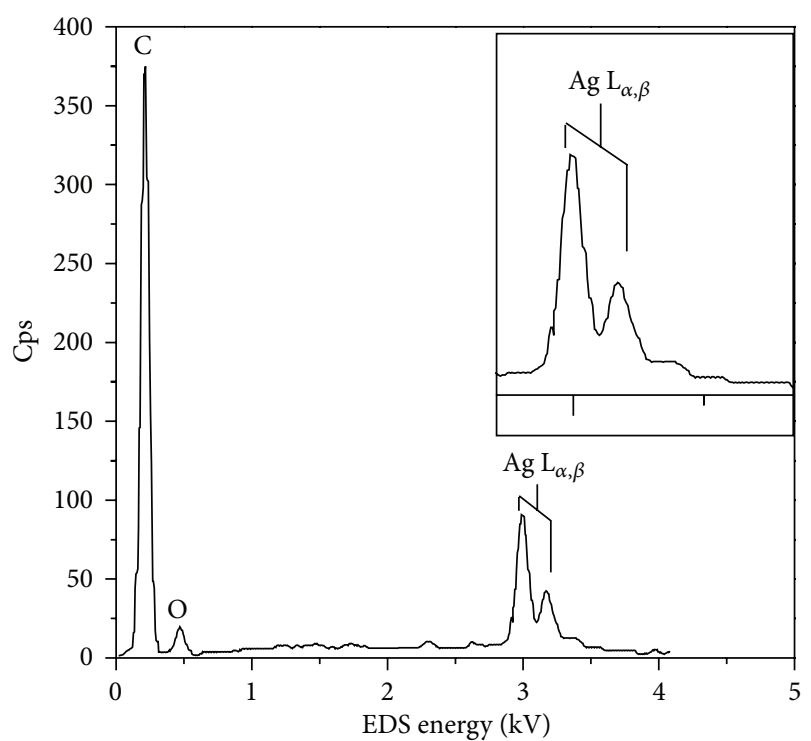

FIGURE 4: EDX spectrum of nylon-6,6 membrane coated with Ag nanoparticles.

located at $390 \mathrm{~nm}$ (Figure 5(a)); this is attributable to the surface plasmon resonance of silver nanoparticles in agreement with the observed yellow colour showed in Figure 3 [19]. No absorption was observed at wavelengths longer than $450 \mathrm{~nm}$. These observations imply that Ag nanoparticles were formed. The surface plasmon peak underwent a shift to

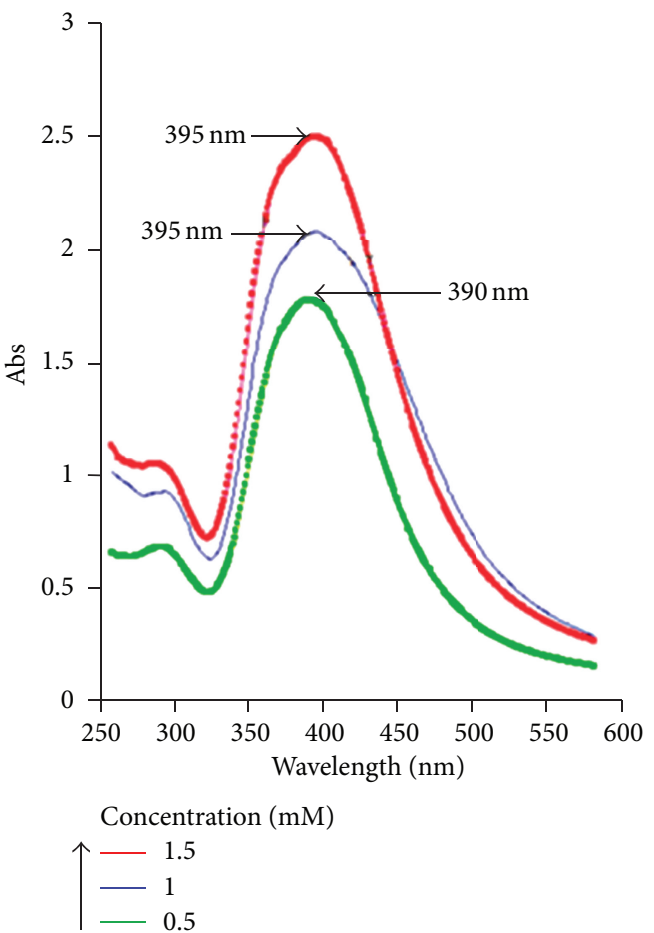

FIGURE 5: UV-Vis spectra of silver nanoparticles in nylon-6,6 membranes. Nanoparticles were prepared using aqueous $\mathrm{AgNO}_{3}$ at $0.5 \mathrm{mM}, 1.0 \mathrm{mM}$, and $1.5 \mathrm{mM}$, respectively. 

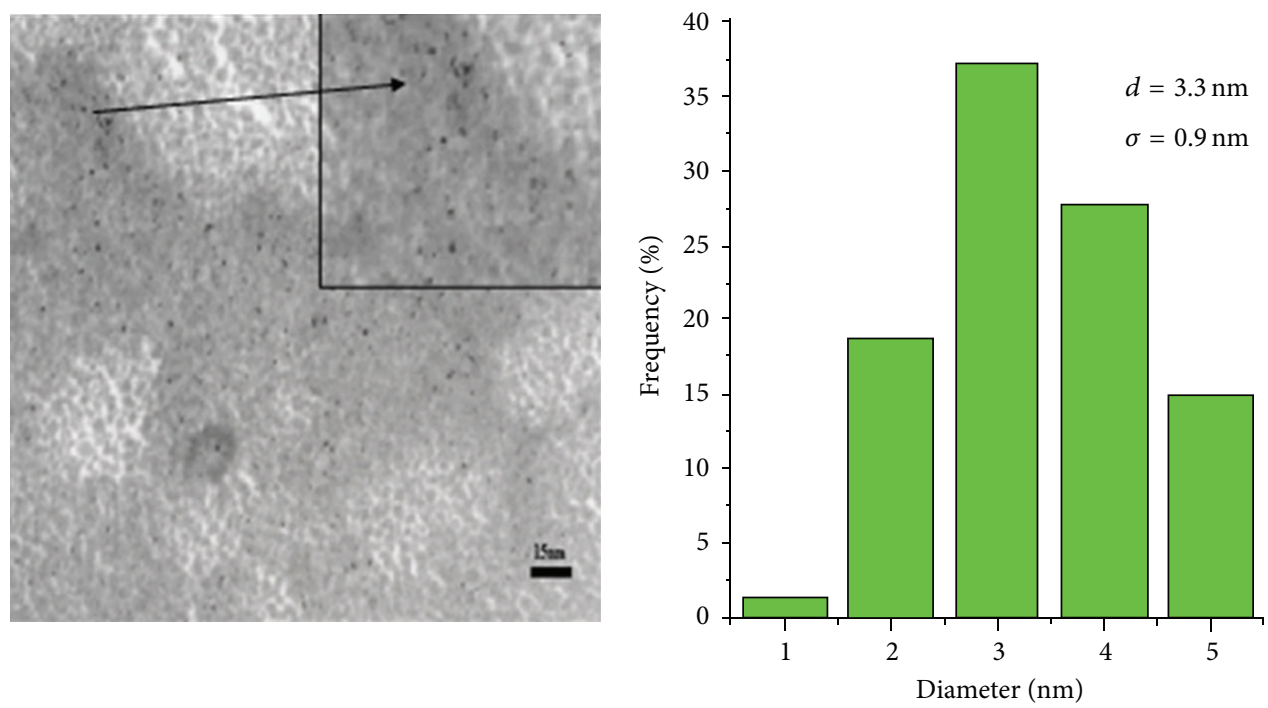

FIGURE 6: TEM micrograph and size distribution histogram of Ag nanoparticles in nylon-6,6 membranes $\left(\mathrm{AgNO}_{3} 0.05 \mathrm{mM}\right)$.

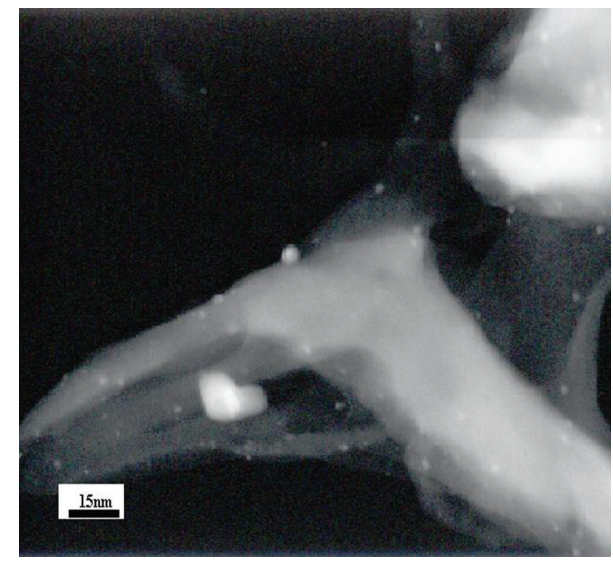

(a)

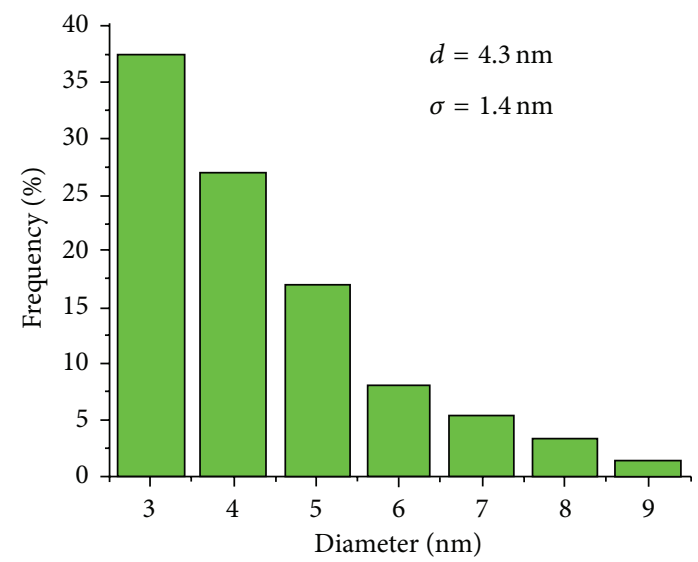

(c)

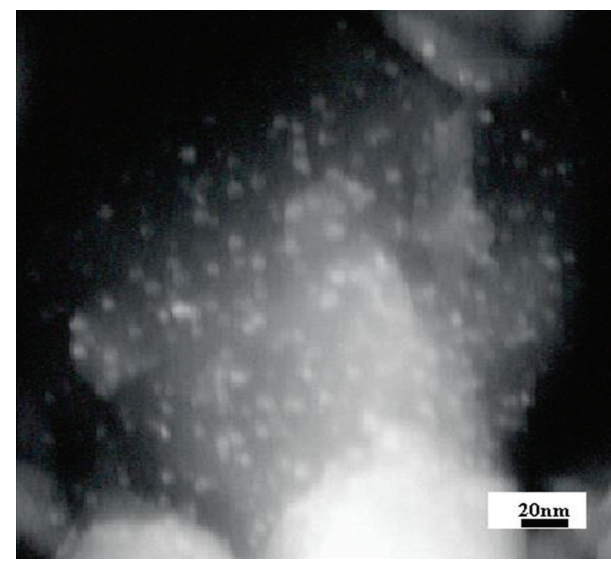

(b)

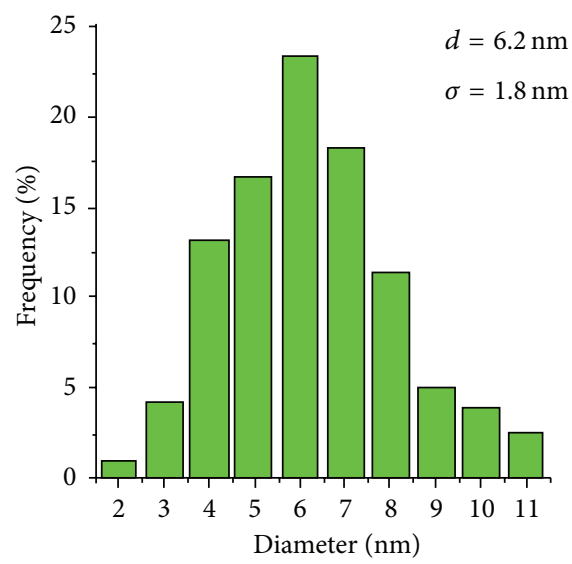

(d)

FIGURE 7: HAADF images and size distribution of Ag nanoparticles obtained from (a), (c) $\mathrm{AgNO}_{3} 1.0 \mathrm{mM}$; (b), (d) $\mathrm{AgNO} 31.5 \mathrm{mM}$. 
$395 \mathrm{~nm}$ and was slightly broadened when $1 \mathrm{mM} \mathrm{AgNO}_{3}$ was employed (Figure 5(b)). When $1.5 \mathrm{mM} \mathrm{AgNO}_{3}$ was used, the surface plasmon absorption becomes broader (Figure 5(c)). It is noted that the absorption intensity of the plasmon band increases as the concentration of aqueous $\mathrm{AgNO}_{3}$ augments, also in agreement with the membrane intensity changes in colour, which can be attributed to the increase in the $\mathrm{Ag}$ nanoparticles concentration.

These assumptions are confirmed by TEM observations. As shown in Figure 6 monodisperse Ag nanoparticles were obtained at $\mathrm{AgNO}_{3} 0.5 \mathrm{mM}$. Their mean diameter (d) was measured as $3.3 \mathrm{~nm}$ with a standard deviation $(\sigma)$ of $0.9 \mathrm{~nm}$.

Figure 7 shows $Z$-contrast images of nylon fibres with $\mathrm{Ag}$ particles. Figures $7(a)$ and $7(c)$ correspond to a concentration of $1.0 \mathrm{mM}$ of $\mathrm{AgNO}_{3}$ resulting in nanoparticles with an average size of $4.3 \mathrm{~nm}$ with a standard deviation of $1.4 \mathrm{~nm}$. Using a solution of $1.5 \mathrm{mM} \mathrm{AgNO}_{3}$ (Figures 7(b) and 7(d)), the average particle size slightly increases to $6.2 \mathrm{~nm}$ with a standard deviation of $1.8 \mathrm{~nm}$. Therefore, it is possible to control the size and size distribution by adjusting the concentration of metal ions in solution.

Because of the porous structure of nylon fibre and the strong interactions between $\mathrm{Ag}^{+}$ions and the carbonyl and amide groups of nylon macromolecule, $\mathrm{Ag}^{+}$ions were uniformly and tightly anchored to the nylon fibres [20]. Such interactions would lower the mobility of $\mathrm{Ag}^{+}$ions, enhance the formation of silver nuclei, limit the formation of several morphologies, and prevent the growth of larger particles [21]. This is particularly true at low $\mathrm{Ag}^{+}$ion concentrations and can explain the formation of monodisperse $\mathrm{Ag}$ nanoparticles (after $\mathrm{NaBH}_{4}$ reduction) under such conditions. At higher $\mathrm{AgNO}_{3}$ concentrations, larger amounts of $\mathrm{Ag}^{+}$ions are embedded on nylon membranes, leading to large and widely distributed particles after reduction [22]. In these kinds of systems, it is expected that the carbonyl and amide groups may also play an important role in stabilization of metal nanoparticles in addition to the porous structure of nylon fibres.

The HRTEM image in Figure 8 shows Ag nanoparticles between 2 and $3 \mathrm{~nm}$ in diameter. This image possesses atomic resolution; therefore, several crystalline planes are distinguishable and the interplanar distances can be measured. The interplanar distances shown in the micrograph were measured from the Fourier transforms (FFT) of these nanoparticles (Figure 8, bottom), where the corresponding crystalline planes are specified. The interplanar distances and their corresponding crystalline planes match the ones of metallic $\mathrm{Ag}$ (FCC) phase. The measured interplanar distance is $2.36 \AA$ and corresponds to the (111) plane [23].

In order to examine the chemical composition of the nylon-6,6/Ag nanoparticles fibre composite, as well as the possible interaction of silver metal with the nylon moieties, after formation of Ag nanoparticles, X-ray photoelectronic spectroscopy (XPS) was used. Figure 9 shows the XPS survey spectra obtained after $\mathrm{Ar}^{+}$etching for $10 \mathrm{~s}$.

Figure 9 shows the increase in the $\mathrm{Ag} 3 \mathrm{~d}$ signal corresponding to the different concentrations of $\mathrm{AgNO}_{3}$ added in the solution reaction; a spectrum of nylon-6,6 is used as reference. Clearly an increase of the concentration of

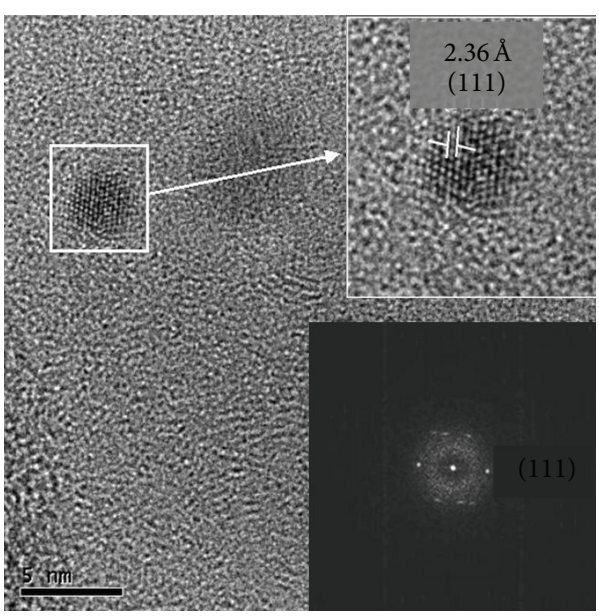

FIGURE 8: HRTEM image of Ag nanoparticles (top) and their corresponding Fourier transforms (FFT) (bottom) pattern.

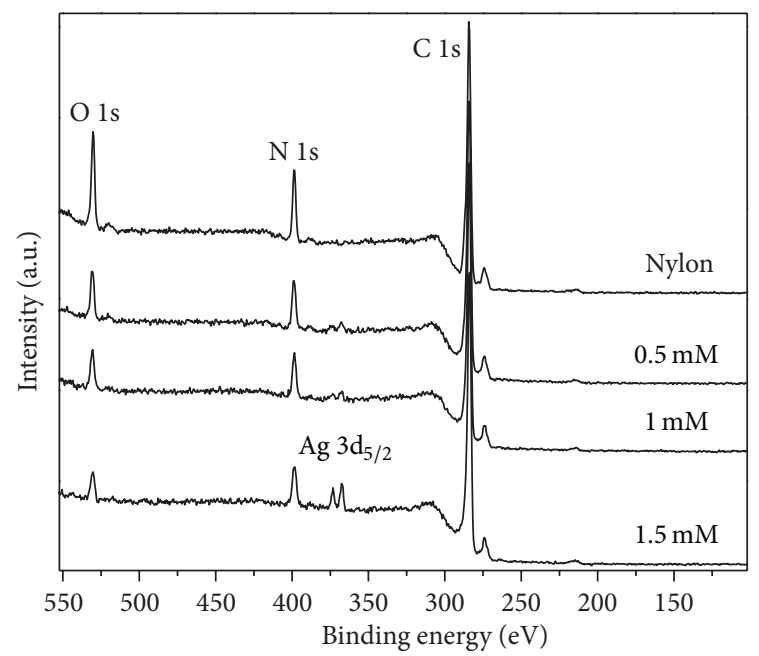

FIGURE 9: XPS spectra of nylon-6,6 and three different nylon-6,6/Ag composites.

the metal precursor salt favours the formation of the nylon/ Ag composite.

In order to obtain more information about the chemical state of the Ag nanoparticles present in the composite a curve fit of the various signals was made and is shown in Figure 10.

Table 1 shows the binding energies of the XPS deconvolution of $\mathrm{Ag} \mathrm{3d}, \mathrm{C} 1 \mathrm{~s}, \mathrm{O} 1 \mathrm{~s}$, and $\mathrm{N}$ 1s core levels samples with the Ag metallic, $\mathrm{AgO}$, and nylon-6,6 as references. The peak energy position in the deconvolution and number of peaks were based in data reported by Beamson and Brigs [24] and Hoflund and Hazos [25] and calibrated with metallic Ag foil as reference.

From Table 1, a positive chemical shift in $\mathrm{N}$ 1s core level and negative chemical shift in $\mathrm{Ag} 3 \mathrm{~d}_{5 / 2}$ of $\mathrm{Ag}(0)$ of nanoparticles, with respect to $\mathrm{N}$ 1s and $\mathrm{Ag} 3 \mathrm{~d}_{5 / 2}$ of nylon and $\mathrm{Ag}$ metallic, are observed. From the data in Table 1 the general rule based on the electronegativity can explain both the positive chemical shift of the $\mathrm{N}$ 1s core level and the negative chemical shift of the Ag $3 d_{5 / 2}$ core level. This effect can be seen 
TABLE 1: The binding energy position by XPS of nylon-6,6- and Ag-containing samples core levels Ag 3d, C 1s, O 1s, and N 1s.

\begin{tabular}{|c|c|c|c|c|c|c|c|c|c|}
\hline \multirow[b]{2}{*}{ Sample } & \multirow{2}{*}{$\mathrm{N} 1 \mathrm{~s}$} & \multicolumn{2}{|c|}{$\mathrm{O} 1 \mathrm{~s}$} & \multicolumn{2}{|c|}{$\operatorname{Ag} 3 d_{5 / 2}$} & \multicolumn{4}{|c|}{${ }^{*} \mathrm{C} 1 \mathrm{~s}$} \\
\hline & & $\mathrm{O}=\mathrm{C}$ & $\mathrm{O}-\mathrm{Ag}$ & Ag & $\begin{array}{l}\mathrm{AgO} \\
(\mathrm{eV})\end{array}$ & 1 & 2 & 3 & 4 \\
\hline References* & 398.69 & 530.33 & & 368.21 & & & & & \\
\hline $0.5 \mathrm{mM}$ & 398.91 & 530.33 & 531.30 & & & & & م050 & ד \\
\hline $1.0 \mathrm{mM}$ & 398.80 & 530.33 & 531.30 & 368.20 & 367.31 & 283.84 & 284.14 & 285.00 & 286.81 \\
\hline $1.5 \mathrm{mM}$ & 398.75 & 530.33 & 531.30 & & & & & & \\
\hline
\end{tabular}

${ }^{*}$ References: nylon and metallic Ag.
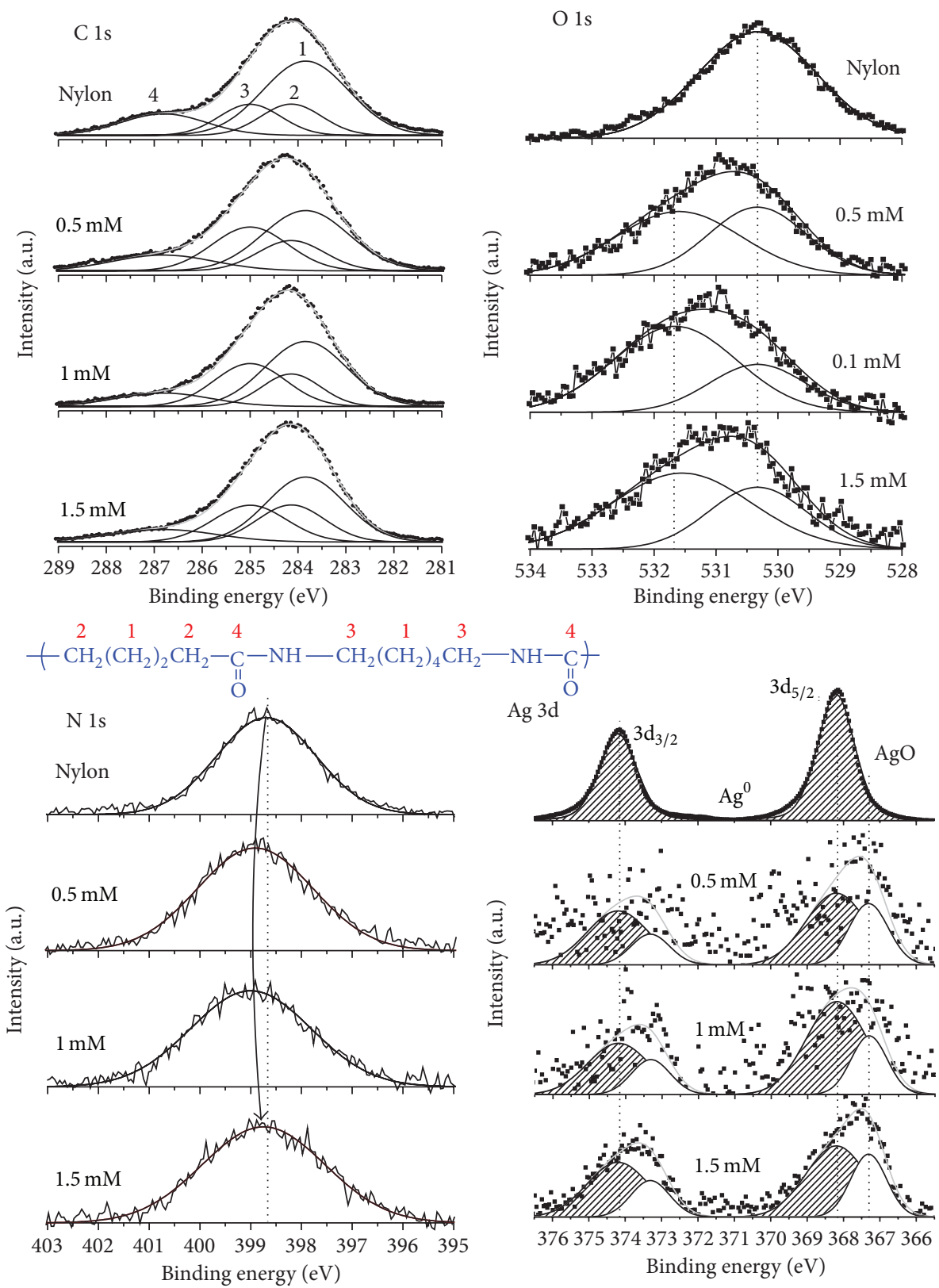

FIgURE 10: The XPS deconvolution for C 1s, O 1s, N 1s, and Ag 3d core levels.

in Figure 10 for $\mathrm{N}$ 1s. For the composite samples analysed, all $\mathrm{C} 1 \mathrm{~s}, \mathrm{O} 1 \mathrm{~s}$, and $\mathrm{Ag} 3 \mathrm{~d}_{5 / 2}$ of AgO core levels are in the same positions as exhibited in Table 1 and Figure 10. $\mathrm{O} 1 \mathrm{~s}$ $(530.33 \mathrm{eV})$ corresponds to $\mathrm{O}=\mathrm{C}$ and $\mathrm{Ag}-\mathrm{O}$ at $367.31 \mathrm{eV} ; \mathrm{C}$ 1s corresponds to (1) $\mathrm{C}-\mathrm{CH}_{2}$ at $283.84 \mathrm{eV}$, (2) $\mathrm{CH}_{2}-\mathrm{CH}_{2}$ at
$284.14 \mathrm{eV}$, (3) $\mathrm{CH}_{2}-\mathrm{N}$ at $285 \mathrm{eV}$, and (4) $\mathrm{C}=\mathrm{O}$ at $286.81 \mathrm{eV}$. It can be also observed that for $\mathrm{N}$ 1s the maximum shifting $(\Delta)$ occurs for the $0.5 \mathrm{mM}$ simple with $\Delta=0.22 \mathrm{eV}$; however, for $0.1 \mathrm{mM}$ sample, $\Delta=0.11$, it contains the largest amount of Ag nanoparticles, taking in account the amount 
of AgO. For the $1.5 \mathrm{mM}$ sample, Ag $3 \mathrm{~d}$ peaks can be deconvoluted satisfactorily obtaining thus all oxidation states even in the samples with less Ag concentration. In addition, in Figure 10, the data obtained from the curve fitting show that there is no change in the binding energy of the orbitals of $\mathrm{C}$ 1s despite the use of different $\mathrm{AgNO}_{3}$ concentrations. There is, however, a $0.31 \mathrm{eV}$ shift in the $\mathrm{N}$ 1s peak corresponding to the sample with $1.5 \mathrm{mM} \mathrm{AgNO}$. This small shift in binding energy suggests there is an interaction of the nitrogen atoms in nylon during the stabilization of the silver nanoparticles. A similar chemical shift for $\mathrm{N}$ 1s binding energy in XPS spectra has been observed for the interactions of PVP with $\mathrm{Pt}$ nanoparticles and nitrocellulose with $\mathrm{Ru}[26,27]$. Hence, pores of nylon fibers, where the nitrogen atoms from the amide groups are found, not only interact with the metal ions presumably through iondipole forces, but once the reduction reaction occurs, they also stabilize the Ag nanoparticles. Analysis of the $\mathrm{O} 1 \mathrm{~s}$ and $\mathrm{Ag} 3 \mathrm{~d}_{5 / 2}$ peaks shows the presence of $\mathrm{AgO}$, which was expected due to the high reactivity of the silver nanoparticles at the sizes obtained. Nevertheless, the fact that there is still a signal corresponding to $\mathrm{Ag}^{0}$ suggests that the oxidation takes place only on the surface of the nanoparticles, while metallic silver remains at their core; again this is possible due to the stabilizing effect of the amide groups present in the nylon fibers. This nylon-6,6/Ag nanoparticles composite will be probed in dyes removal from aqueous solutions and in applications regarding its antibacterial properties.

\section{Conclusions}

It was demonstrated that using an aqueous Ag ion impregnation of nylon fibres followed by a reduction with $\mathrm{NaBH}_{4}$, a composite of $\mathrm{Ag}$ nanoparticles attached to the polymer can be formed. The whole process is carried out at ambient conditions. SEM, HRTEM, and XPS studies confirmed the presence of such Ag nanoparticles in the fibres, with an average size of $3.3 \mathrm{~nm}$. This very simple and versatile synthetic route could be applied to obtain other composites made of metal nanoparticles and natural or synthetic polymer fibres. Moreover, an interaction between nitrogen of amides moieties of nylon-6,6 and silver nanoparticles has been found by X-ray photoelectron spectroscopy.

\section{Acknowledgments}

Financial support of this work was provided by Universidad Autónoma del Estado de México (UAEM) (Project no. $3246 / 2012 \mathrm{U})$. The authors are indebted to Fernando Ureña (ININ), Samuel Tehuacanero (IFUNAM) for their assistance in SEM and image digitalisation, respectively.

\section{References}

[1] J. Y. Ying, C. P. Mehert, and M. S. Wong, "Synthesis and applications of supramolecular-templated mesoporous materials," Angewandte Chemie, vol. 38, no. 1-2, pp. 56-77, 1999.

[2] M. S. Morey, J. D. Bryan, S. Schartz, and G. D. Stuchy, "Pore surface functionalization of MCM-48 mesoporous silica with tungsten and molybdenum metal centers: perspectives on catalytic peroxide activation," Chemistry of Materials, vol. 12, no. 11, pp. 3435-3444, 2000.

[3] J. Liu, X. Feng, G. E. Fryxell, L. Wang, A. Y. Kim, and M. Gong, "Hybrid mesoporous materials with functionalized monolayers," Advanced Materials, vol. 10, no. 2, pp. 161-165, 1998.

[4] J. J. Schneider, N. Czap, J. Hagen et al., "Metallorganic routes to nanoscale iron and titanium oxide particles encapsulated in mesoporous alumina: formation, physical properties, and chemical reactivity," Chemistry, vol. 6, no. 23, pp. 4305-4321, 2000.

[5] J. S. Jung, W. S. Chae, R. A. McIntyre, C. T. Selp, J. B. Wiley, and C. J. O'Connor, "Preparation and characterization of $\mathrm{Ni}$ nanoparticles in an MCM mesoporous material," Materials Research Bulletin, vol. 34, no. 9, pp. 1353-1360, 1999.

[6] K. Moller and T. Bein, "Inclusion chemistry in periodic mesoporous hosts," Chemistry of Materials, vol. 10, no. 10, pp. 29502963, 1998.

[7] H. Shi, L. Zhang, and W. Cal, "Composition modulation of optical absorption in $\mathrm{Ag}_{x} \mathrm{Au}_{1-x}$ alloy nanocrystals in situ formed within pores of mesoporous silica," Journal of Applied Physics, vol. 87, no. 3, p. 1572, 2000.

[8] K. W. Powers and L. L. Hench, "The effect of pore size on metal cluster formation in silica sol gel monoliths," Ceramic Transactions, vol. 101, p. 253, 2000.

[9] Y. Plyuto, J. M. Berquer, C. Jaquiod, and C. Ricolleau, "Ag nanoparticles synthesised in template-structured mesoporous silica films on a glass substrate," Chemical Communications, no. 17, pp. 1653-1654, 1999.

[10] W. H. Zhang, J. L. Shi, L. Z. Wang, and D. S. Yan, "Preparation and characterization of $\mathrm{ZnO}$ clusters inside mesoporous silica," Chemistry of Materials, vol. 12, no. 5, pp. 1408-1413, 2000.

[11] B. Lebeau, C. E. Fowler, S. Mann, C. Farcet, B. Charleux, and C. Sanchez, "Synthesis of hierarchically ordered dyefunctionalised mesoporous silica with macroporous architecture by dual templating," Journal of Materials Chemistry, vol. 10, no. 9, pp. 2105-2108, 2000.

[12] L. Zhang, T. Sun, and J. Y. Ying, "Oxidation catalysis over functionalized metalloporphyrins fixated within ultralarge-pore transition metal-doped silicate supports," Chemical Communications, no. 12, pp. 1103-1104, 1999.

[13] V. L. Colvin, M. C. Schlamp, and A. P. Alivisatos, "Light-emitting diodes made from cadmium selenide nanocrystals and a semiconducting polymer," Nature, vol. 370, no. 6488, pp. 354357, 1994.

[14] B. O. Dabbousi, M. G. Bawendi, O. Onitsuka, and M. F. Rubner, "Electroluminescence from CdSe quantum-dot/polymer composites," Applied Physics Letters, vol. 99, p. 13834, 1995.

[15] R. Lamber, S. Wetjen, G. Schulz-Ekloff, and A. Baalmann, "Metal clusters in plasma polymer matrices: gold clusters," Journal of Physical Chemistry, vol. 99, no. 38, pp. 13834-13838, 1995.

[16] L. L. Beecroft and C. K. Ober, "Nanocomposite materials for optical applications," Chemistry of Materials, vol. 9, no. 6, pp. 1302-1317, 1997.

[17] K. Akamatsu, N. Tsuboi, Y. Hatakenaka, and S. Deki, "In situ spectroscopic and microscopic study on dispersion of $\mathrm{Ag}$ nanoparticles in polymer thin films," Journal of Physical Chemistry B, vol. 104, no. 44, pp. 10168-10173, 2000.

[18] SDP v4. 1 (32 bit) Copyright 2004, XPS International, LLC, Compiled January 17, 2004. 
[19] J. He, I. Ichinose, T. Kunitake, and A. Nakao, "Reversible conversion of nanoparticles of metallic silver and silver oxide in ultrathin $\mathrm{TiO}_{2}$ films: a chemical transformation in nano-space," Chemical Communications, no. 17, pp. 1910-1911, 2002.

[20] S. Besson, T. Gacoin, C. Ricolleau, and J. Boilot, "Silver nanoparticle growth in 3D-hexagonal mesoporous silica films," Chemical Communications, vol. 9, no. 3, pp. 360-361, 2003.

[21] T. M. Fahmy, P. M. Fong, A. Goyal, and W. M. Saltzman, “Targeted for drug delivery," Nanotoday, vol. 8, no. 8, pp. 18-26, 2005.

[22] J. He, I. Ichinose, T. Kunitake, A. Nakao, Y. Shiraishi, and N. Toshima, "Facile fabrication of Ag-Pd bimetallic nanoparticles in ultrathin $\mathrm{TiO}_{2}$-gel films: nanoparticle morphology and catalytic activity," Journal of the American Chemical Society, vol. 125, no. 36, pp. 11034-11040, 2003.

[23] W. J. de Ruijter, R. Sharma, M. R. McCartney, and D. J. Smith, "Measurement of lattice-fringe vectors from digital HREM images: experimental precision," Ultramicroscopy, vol. 57, no. 4, pp. 409-422, 1995.

[24] G. Beamson and D. Brigs, High Resolution XPS of Organic Polymers, John Wiley \& Sons, Chichester, UK, 1992.

[25] G. B. Hoflund and Z. F. Hazos, "Surface characterization study of $\mathrm{Ag}, \mathrm{AgO}$, and $\mathrm{Ag}_{2} \mathrm{O}$ using x-ray photoelectron spectroscopy and electron energy-loss spectroscopy," Physical Review B, vol. 62, no. 16, pp. 11126-11133, 2000.

[26] L. Qiu, F. Liu, L. Zhao, W. Yang, and J. Yao, "Evidence of a unique electron donor-acceptor property for platinum nanoparticles as studied by XPS," Langmuir, vol. 22, no. 10, pp. 4480-4482, 2006.

[27] V. K. Kaushik, "XPS core level spectra and Auger parameters for some silver compounds," Journal of Electron Spectroscopy and Related Phenomena, vol. 56, no. 3, pp. 273-277, 1991. 

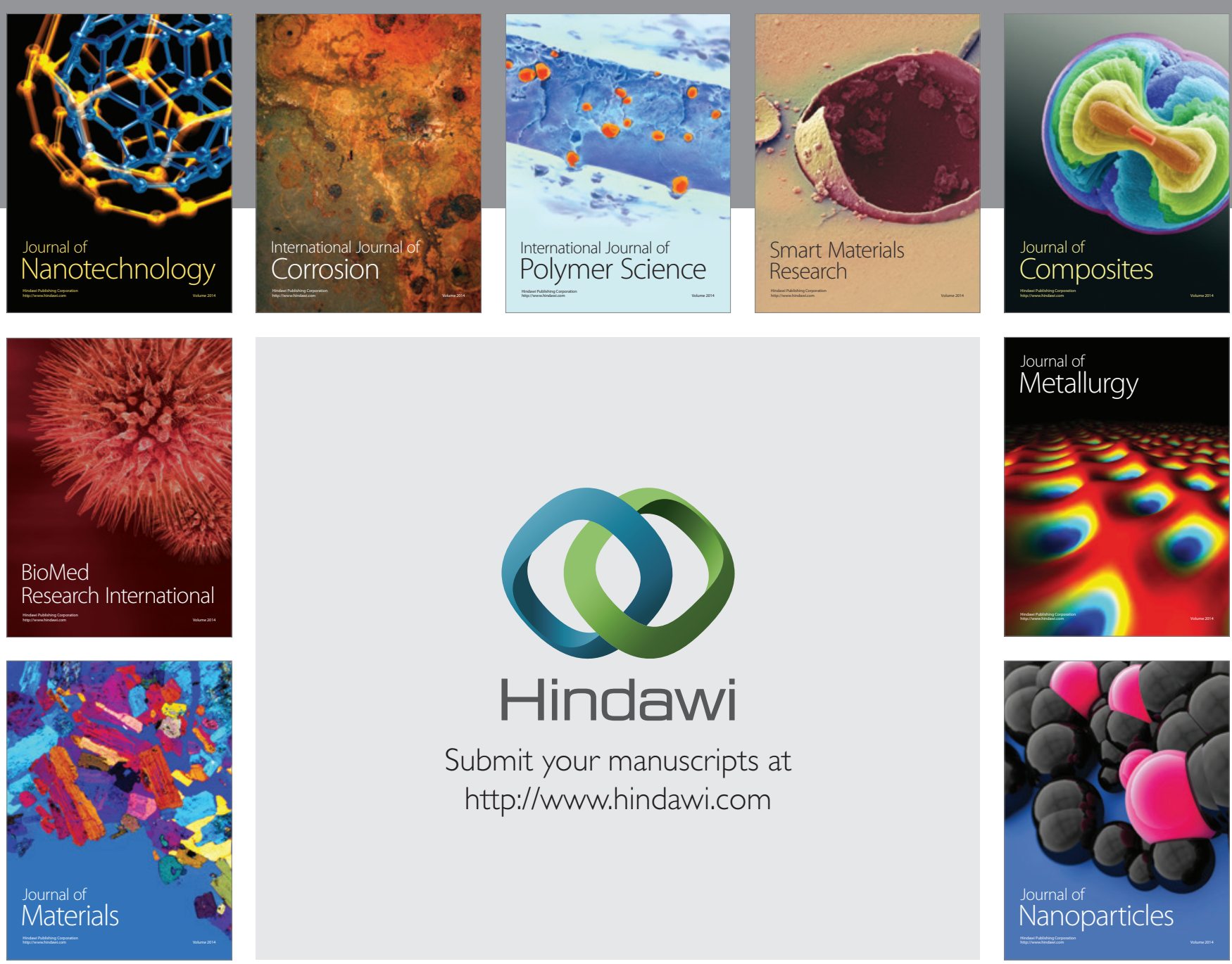

Submit your manuscripts at http://www.hindawi.com
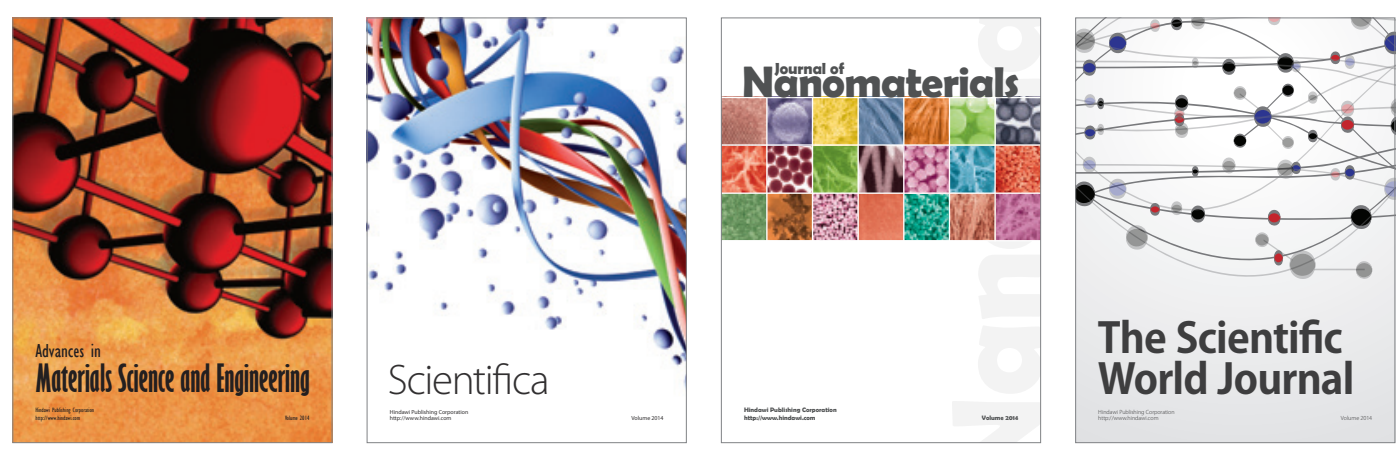

\section{The Scientific World Journal}
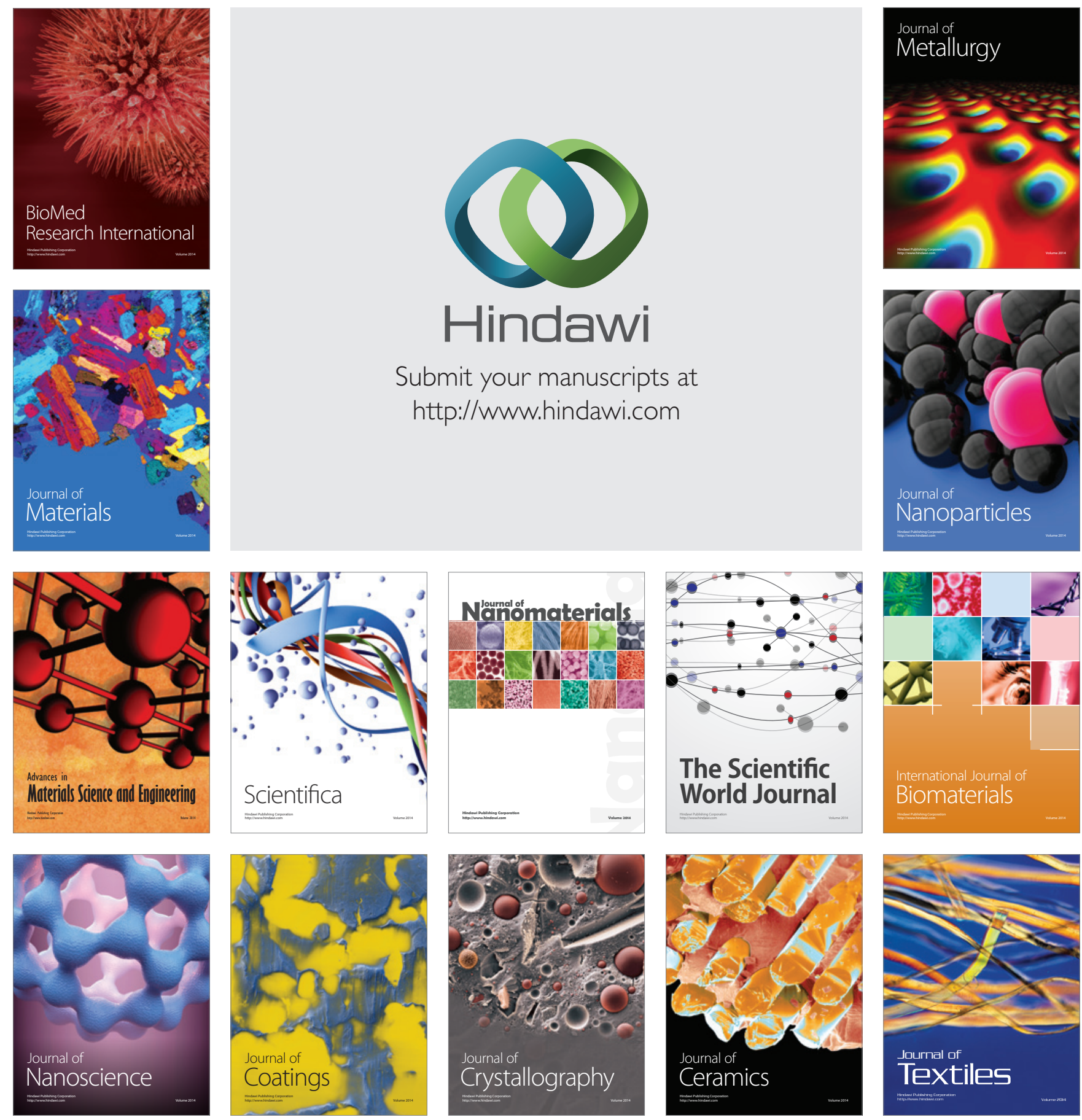